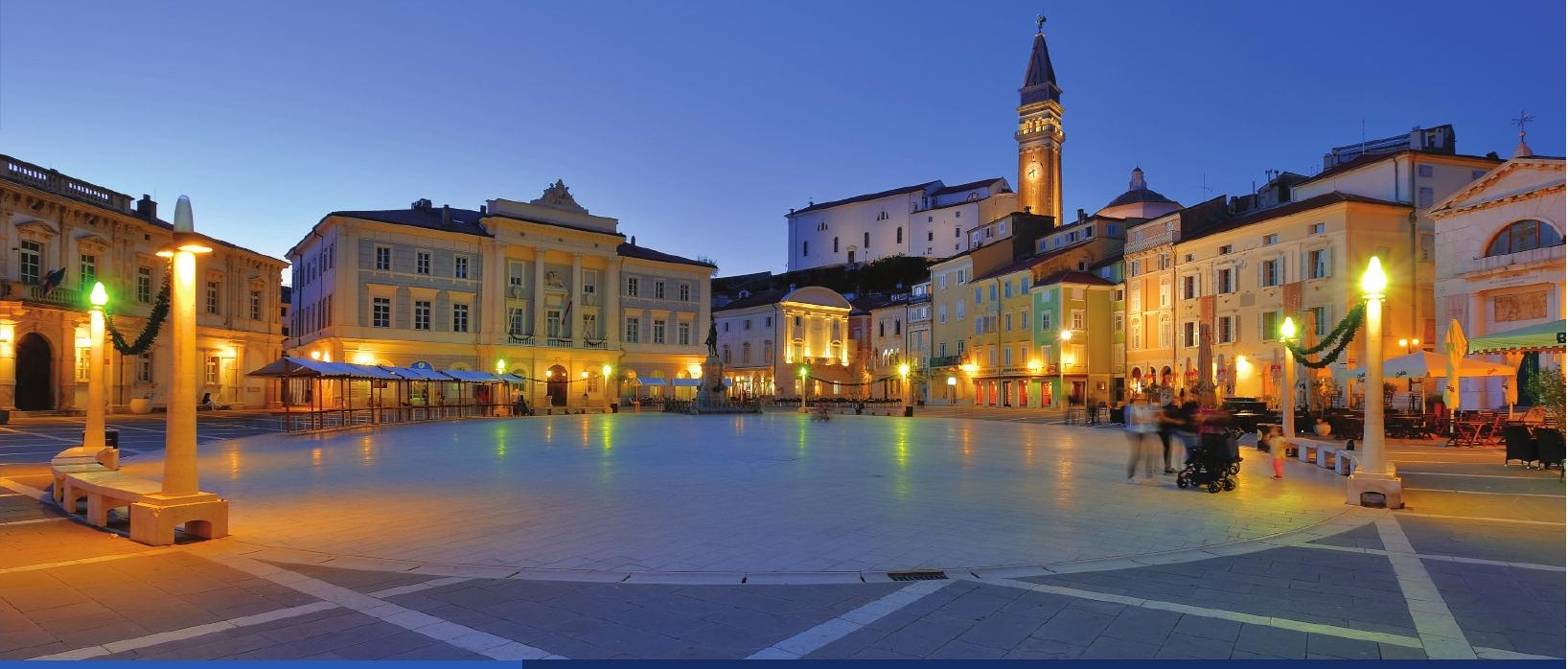

-- Intuition, Imagination and Innovation

in Suicidology

Conference

Virtual Conference 16th-19th June 2020

Programme and Abstract Book 

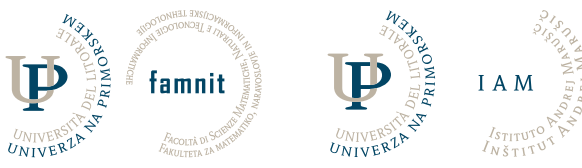

Slovenski

\section{A S $\mathcal{P}$ deleofund}

\section{zer \\ Suicide Because \\ ONE life lost \\ Alliance is ONE too many}

Intuition, Imagination and Innovation in Suicidology Conference 11th Triple i | Virtual Conference | 16th-19th June 2020

Programme and Abstract Book

E-ISSN 2712-5467

Edited by - Diego de Leo and Vita Poštuvan

Cover Photo, Design and Layout · Alen Ježovnik

Published by · University of Primorska press

Titov trg 4, 6000 Koper

www.hippocampus.si

Editor in Chief · Jonatan Vinkler

Managing Editor · Alen Ježovnik

Koper $\cdot 2020$

(C) 2020 University of Primorska

\section{Electronic Edition}

http://www.hippocampus.si/ISBN/978-961-7055-67-2.pdf

http://www.hippocampus.si/ISBN/978-961-293-0oo-4/index.html

https://doi.org/10.26493/978-961-7055-67-2

\section{(cc) BY-SA}

Kataložni zapis o publikaciji (CIP) pripravili

v Narodni in univerzitetni knjižnici v Ljubljani

COBISS.SI-ID $=20910851$

ISBN 978-961-7055-67-2 (pdf)

ISBN 978-961-293-000-4 (html) 


\section{Table of Contents}

Welcome Address · 4

Organizers $\cdot 5$

Programme Outline $\cdot 7$

Listening to the Suicidal in the Wake of Covid-19

Vanda Scott $\cdot 8$

Are There Some Premature Deaths We Should Not Prevent? Suicide Prevention When Assistance in Dying is Available

Brian L. Mishara $\cdot 9$

Suicide Prevention at Scale:

The Twin Challenges of Implementation and Evaluation

Navneet Kapur $\cdot 10$

Self-Harm in Children and Adolescents: An Update on Key Issues

Keith Hawton 11

Community Interventions

Lakshmi Vijayakumar 13

Impacts of Covid-19 on Mental Health and Suicide Risk in Occupational Settings:

Implications for Suicide Prevention and Research

Ella Arensman $\cdot 14$

Covid-19 and Suicide Prevention

Diego De Leo 16

Zero Suicide: A Big Hairy Audacious Goal

Joe Rafferty $\cdot 17$ 


\section{Welcome Address}

Dear intuitive, imaginative and innovative guest,

We are happy to welcome you at the Triple i Virtual Conference, event this year brought to you by Slovene Centre for Suicide Research, De Leo Fund Onlus and International Association for Suicide Prevention!

The conference is an international annual event organized by the Slovene Centre for Suicide Research (Andrej Marušič Institute, University of Primorska) in collaboration with FAMNIT (University of Primorska). Our aim is to increase awareness and knowledge about suicide and thus contribute to the development of suicide prevention and postvention activities.

In recent years, the conference has developed into a platform for fruitful discussions among distinguished experts in suicide research and prevention, interacting with young and perspective suicidologists and other mental health professionals.

Regrettably, due to the epidemiological situation regarding the spread of the SARS-CoV-2 virus, we had to cancel this year's Triple i conference. Nonetheless, employees from different organizations connected and prepared a virtual version of the traditional conference, as we want to enable eager participants to listen to and interact with world-renowned suicidologists. The exchange of knowledge and experience regarding suicidal behaviour is all the more important in these new and challenging circumstances. And attendance at the virtual conference thus enables just that: the opportunity to listen to new and innovative findings in the field of suicidology while also connecting and exchanging knowledge with participants from countries around the world.

The Triple i 2020 programme includes topics of suicide research and clinical work with suicidal patients and thus addresses theory, research, prevention and interventions in suicidology. Each lecture will be followed by a discussion in which the participants will be invited to share their views. Existing difficulties in both research and clinical work will be specifically addressed. Let the Triple i Conference be an opportunity for you to express and practice your intuition, imagination and innovative ideas.

Prof. Diego De Leo and Dr. Vita Poštuvan, Chairs

Wendy Orchard, Wendy Cliff, Vanja Gomboc, and Katherine Thomson, Organising Committee 


\section{Organizers}

\section{Slovene Centre for Suicide Research (UP IAM SCSR)}

Slovene Centre for Suicide Research was founded in 2011 within UP Andrej Marušič Institute, University of Primorska. The initiative for establishment of an independent unit within the institute was put forward by Prof. Diego De Leo and a group of former co-workers of late Prof. Andrej Marušič in the memory of his work and with the purpose of continuing it.

Research and prevention of suicide are addressed as crucial activities of clinical-research work. The Centre is involved in international and national projects and is the initiator of numerous innovative approaches in suicidology. The Centre's vision is to provide high-quality research for better understanding of suicidal behaviour, thus contributing to suicide prevention, intervention and postvention.

You are welcome to read more about us at zivziv.si!

\section{De Leo Fund Onlus}

De Leo Fund Onlus was established with the desire to offer a source of support for parents, children, relatives and friends who are suddenly forced to face the death of a loved one, emerging from road accidents, work-related accidents, suicide, natural disasters, crimes. At such times, human and professional support can play a role of enormous significance in mitigating pain and isolation of which invariably suffer the survivors to these deaths.

In parallel with care activities, De Leo Fund organizes training courses, meetings and seminars. In the context of scientific projects, De Leo Fund collaborates with universities and other institutions to carry out research programs and educational activities in the field of suicide and traumatic bereavement issues in children, adolescents and adults.

You are welcome to read more about us at https://www.deleofund.org!

The International Association for Suicide Prevention (IASP)

The International Association for Suicide Prevention (IASP) is a Non-Governmental Organization and the largest international organization dedicated to suicide prevention. It was founded by the late Professor Erwin Ringel and Dr. Norman Farberow in 1960 and now includes professionals and volunteers from 77 countries. 
The International Association for Suicide Prevention (IASP) is dedicated to preventing suicidal behaviour, alleviating its effects, and providing a forum for academics, mental health professionals, crisis workers, volunteers and suicide survivors.

You are welcome to read more about us at https://www.iasp.info! 


\section{Programme Outline}

Tuesday, 16th June 2020

10.00-11.00 Ms. Vanda Scott, 'Listening to the Suicidal in Wake of Covid-19.'

16.00-17.00 Prof. Brian L. Mishara, 'Are There Some Premature Deaths We Should Not Prevent? Suicide Prevention When Assistance in Dying is Available.'

Wednesday 17th June 2020

10.00-11.00 Prof. Navneet Kapur, 'Suicide Prevention at Scale: The Twin Challenges of Implementation and Evaluation.'

16.00-17.00 Prof. Keith Hawton, 'Self-Harm in Children and Adolescents: Key Issues.'

Thursday 18th June 2020

10.00-11.00 Dr. Lakshmi Vijayakumar, 'Community Interventions.'

16.00-17.00 Prof. Ella Arensman, 'Impacts of Covid-19 on Mental Health and Suicide Risk in Occupational Settings: Implications for Suicide Prevention and Research.'

Friday 19th June 2020

10.00-11.00 Prof. Diego De Leo, 'Covid-19 and Suicide Prevention.'

16.00-17.00 Mr. Joe Rafferty, 'Zero Suicide: A Big Hairy Audacious Goal.' 


\section{Listening to the Suicidal in the Wake of Covid-19}

Vanda Scott

Ms. Vanda Scott is currently the international advisor to the International Association for Suicide Prevention and holds several advisory positions with NGOs including the De Leo Fund. She participated in the development of a worldwide network of agencies for suicide prevention, which enhances cooperation between mental health professionals and volunteers. Her speech will be about cooperative approaches to prevention of suicide with examples on three levels - local, national and international.

Abstract. Unknown, unexpected, medical emergency - Covid-19 a global crisis. In December 2019 coronavirus was identified in China and by early March extended exponentially across many nations, East and West, and the World Health Organisation declared Covid-19, a global pandemic. Globally, governments immediately enforced a range of actions from the research of the virus through to extensive medical treatment, social isolation, workplace and countrywide closures. The impact is unmeasurable and will affect economic and social stability as well as the general and mental health of the population in all countries. Almost $80 \%$ of all suicides occur in the low and middle income countries; these very same countries that will experience even greater inequities during this pandemic due to weak public health systems, lack of mental health professionals and facilities, the negative economic effect of lockdowns and the evident increase in poverty. Along with mental health professionals worldwide, Suicide Prevention Crisis Centres have been in the front line of responding to the public and, unquestionably, will continue to have a significant role in being accessible for those suffering suicidal ideation and behaviour. The purpose of this presentation is to take a small sample of the work from crisis centres in both the developed and developing countries and review the changes in their services that is currently being experienced and also reflect on what could be expected. It is too early to predict the changes in suicidal behaviour. However, this sector is only too aware that there is a need to prepare for an undetermined change in trends in suicidal behaviour along with unknown knowledge on how such changes may be resourced. A few challenging options will be explored. 


\section{Are There Some Premature Deaths We Should Not Prevent? Suicide Prevention When Assistance in Dying is Available}

Brian L. Mishara

Brian Mishara is Professor of Psychology and Director of the Centre for Research and Intervention on Suicide and Euthanasia (CRISE) at the Université du Québec à Montréal, Canada. He is a founder of Suicide Action Montreal, the Montreal suicide prevention centre, a Vice-chairperson of the Trustees of Befrienders Worldwide, an international organization of helplines, a past president of the International Association for Suicide Prevention and Canadian Association for Suicide Prevention. He consults and conducts suicide prevention training internationally and has helped establish helplines using telephone, chat and text services. As he likes challenges, he is an active researcher in several fields, among which we should mention the effectiveness of suicide prevention programmes, understanding suicide in children, theories of suicidality, euthanasia etc. His recent research also focuses on best practices and ethical issues in using new technologies in suicide prevention.

Abstract. Suicide prevention services are generally supposed to do their utmost to prevent suicides with all persons, regardless of the suicidal individual's characteristics and reasons given for wanting to die. Their assumptions are that doing otherwise constitutes discrimination and that they would venture into an ethical morass if they attempt to determine whether some lives are more worthy of saving than others. However, where MAiD (assisted suicide and euthanasia) has been legalized, should we continue to strive to prevent all suicides? Or are there some circumstances where we should abstain from preventing a death by suicide or even encourage people to seek to end their lives by MAiD? This presentation explores if there are justified distinctions between how to respond to people requesting or considering requesting $\mathrm{MAiD}$, and how to respond to suicidal individuals. We examine whether suicide is sometimes rational and without ambivalence, as well as how respect for autonomy may be balanced against obligations to protect vulnerable populations. 


\section{Suicide Prevention at Scale: The Twin Challenges of Implementation and Evaluation}

Navneet Kapur

Prof. Navneet Kapur Professor of Psychiatry and Population Health at the University of Manchester, UK and an Honorary Consultant Psychiatrist at Greater Manchester Mental Health NHS Foundation Trust. His research mostly targets epidemiological methods, the aetiology and treatment of suicide behaviour, as well as patient safety. His main contributions have been focussed in the area of health service responses to suicide. Prof. Kapur is particularly enthusiastic about the research work, because, even though clinical work can be very rewarding, research offers the possibility to impact on the lives of many people not just the person in front of you.

Abstract. Despite decades of research examining the epidemiology and aetiology of suicide we are light on research which tells what works for who. On top of that there is an implementation gap where the things we know might help fail to be adopted into routine practice. In this talk, I will discuss a number of strategies that could improve implementation of our research findings: carrying out the highest quality research to inform practice; messaging it simply; engaging with policy makers and the media; disseminating widely using different methods; supporting staff by developing training and implementation tools; implementing at both national and local levels; and of course persistence. I will then go on to discuss examples of large scale evaluations of suicide prevention strategies and policies before describing a major national initiative from the UK. This is ongoing and takes a phased approach to prevention led by local health areas themselves. The audience will be invited to consider potential challenges and solutions. 


\section{Self-Harm in Children and Adolescents: An Update on Key Issues}

Keith Hawton

Prof. Keith Hawton is a Director of the Centre for Suicide Research at the University of Oxford. For more than 40 years he and his research group have been conducting investigations concerning the causes, treatment, prevention and outcome of suicidal behaviour. Prof. Hawton has received research awards from all the major international suicide prevention organisations. His interests include epidemiology and clinical management of self-harm, suicide and self-harm in adolescents, media influences on self-harm and evaluation of suicide prevention initiatives. Key factors that motivate him to continue working in the field of suicidology are the emergence of new challenges and phenomena, such as the role of the internet in suicidal behaviour, new types of suicidal behaviour; the satisfaction of making contributions to prevention of suicide; rewarding collaborations with a wide range of people, both nationally and internationally; and the fact that the field attracts many people who share the same values.

Abstract. Evidence from several countries highlights increasing rates of both self-harm and suicide in young people. In England for example, rates of self-harm have recently increased very substantially in female adolescents, especially those aged under 17 years of age. Also rates of suicide have increased in under-20 year-olds of both genders over the past decade. As in adults, self-harm is an important risk factor for future suicide. There is a pressing need therefore to identify contributory factors that may assist in the design of prevention and treatment initiatives to try to reduce this phenomenon and to help ameliorate its consequences for young people. This has become all the more pressing with the many difficulties presented by the Covid-19 pandemic. In this presentation the epidemiology of self-harm and suicide will be discussed in the context of the iceberg model. Some key factors that contribute to self-harm will then be considered. These include evidence about the important role of puberty in the onset of self-harm, and the contribution of psychiatric disorders, mood instability and sleep disorders. Self-harm and suicide in children and adolescents often appears to spread between individuals, with clusters of the behaviour being far more common than in adults. While media influences on self-harm and suicide continue to be important in young people, the internet and social networking are making an increasing 
contribution, with some positive aspects, as well as negative ones. Recent findings on the association between self-harm and subsequent suicide will be presented, highlighting the long-term risk of suicide in children and adolescents and the fact that there is often a change of method between acts of self-harm and eventual suicide. The impact of self-harm on families will be considered. The presentation will conclude with a summary of key challenges for prevention, including a discussion of the issue posed by the Covid-19 pandemic. 


\section{Community Interventions}

Lakshmi Vijayakumar

Dr. Lakshmi Vijayakumar is the Head of SNEHA Suicide prevention Centre in Chennai. She is particularly interested in the combination of clinical and public health interventions and suicide prevention possibilities within them. She has been conferred 'Honorary Fellowship' of the Royal College of Psychiatrists (FR(Psych), UK for her work on suicide prevention locally, nationally and globally. She is the first woman psychiatrist from South East Asia to receive this honour. Dr. Vijayakumar was a guest of the Triple i conference for the first time in 2014 when she spoke about the relevance of formulating locally relevant, culturally appropriate, costeffective and sustainable interventions.

Abstract. Suicide rates vary widely across countries and within regions in each country. Developing a 'one size fits all' suicide prevention programmes are unlikely to bring down the suicide rates. A bottoms up reach is also needed. It is crucial to develop cost effective and culturally appropriate interventions involving the community. The major challenges in developing such interventions are identifying and understanding the sociocultural context of the community, engaging with different sections of the community without compromising evidence base or effectiveness of suicide interventions, task shifting and ensuring sustainability. Examples of community suicide prevention interventions from high, middle and low-income countries and for vulnerable sections of the community will be discussed. 


\section{Impacts of Covid-19 on Mental Health and Suicide Risk in Occupational Settings: Implications for Suicide Prevention and Research}

Ella Arensman

Prof. Ella Arensman is Research Professor with the School of Public Health, University College Cork and Chief Scientist with the National Suicide Research Foundation (NSRF), Ireland. She is Vice President of the European Alliance Against Depression, and past President of the International Association for Suicide Prevention. She is also Visiting Professor with the Australian Institute for Suicide Research and Prevention, Griffith University, Brisbane. She has been involved in research and prevention of suicide, self-harm and related issues for more than 30 years, with emphasis on risk and protective factors associated with suicide and self-harm, and effectiveness of preventive programmes. In Ireland, she played a key role in developing the first and second National Suicide Prevention Programme: Reach Out, 2005-2014, and Connecting for Life, 2015-2020.

Abstract. The impacts of the Covid-19 pandemic are far reaching and long lasting for people in many work settings, and especially for those in Small and Medium Enterprises (SMEs) in terms of people's wellbeing and mental health and the social and economic impacts. A review of the impacts of Covid-19 and associated movement restriction measures on people with psychiatric disorders and non-clinical mental health difficulties in occupational settings has been conducted by the MINDUP (Mental Health Promotion and Intervention in Occupational Settings) consortium. Considering the short-term and long-term impacts of Covid-19, including staff stress and anxiety, reduced quality and perhaps intensity of prevention of and care for those with mental disorders, or other impacts associated with the Covid-19-crisis, the MINDUP project is timely while it offers to SMEs support and intervention tools with a specific focus on alleviating depression and anxiety among staff. The reaction to the Covid-19 pandemic led to several changes in occupational settings such as increased and changed emotional work demands for some professionals, e.g. health care workers; exposure to infected patients and remote working. While many large organizations usually support their staff with Employee Assistance Programmes or workplace health promotion 
to support their employees in times of crisis, this is commonly not the case in SMEs and therefore the impact of Covid-19 may be exacerbated in these settings. In addition, loss of employment and financial stressors are wellrecognised risk factors for suicide. Governments should therefore provide financial safety nets, and consideration must be given not only to individuals' current situations but also their futures. For example, many young people have had their education interrupted and are anxious about their prospects. Educational institutions must seek alternative ways. The MINDUP project began in January 2020 and aims to improve mental health and wellbeing in the workplace by developing, implementing and evaluating a multilevel intervention targeting both prevalent psychiatric disorders (depression, anxiety disorders) and non-clinical aspects of mental health (stress, burnout, wellbeing, depressive symptoms). In addition, evidence-based tools and interventions will presented that can be included in response plans, implemented in SMEs and other occupational settings. 


\title{
Covid-19 and Suicide Prevention
}

\author{
Diego De Leo
}

Prof. Diego De Leo (the head of Slovene Centre for Suicide Research and professor of Psychiatry at the Griffith University in Brisbane, Australia), is considered as one of top five world leading experts in suicidology by international professional and scientific public. His research expertise includes definitional issues in suicidology, culture and suicide, international trends and national suicide prevention programs. He received numerous world renowned awards for his scientific work. His bibliography includes over 420 peer-reviewed articles and 180 book chapters.

Abstract. The SARS-CoV-2 pandemic - which causes Covid-19 - is feared to cause a confluence of risk factors capable of increasing suicidal behaviours. Policies to combat infection, such as social distancing, social isolation and quarantine, can have negative effects on vulnerable people such as the elderly and those with mental illness. The increased sense of disconnection from society, physical estrangement and the loss of the usual social and work opportunities can provoke feelings of loneliness and social isolation, which are well-known risk factors for suicide, especially in late life. Quarantine itself has been associated with psychological distress, especially when prolonged, as demonstrated by the previous SARS outbreak. Rationing of resources, frustration and boredom, fear of infection, insufficient or contradictory information are all stressful factors associated with poor mental health outcomes. Anxiety, depression and stress-related disorders are common consequences of emergency situations, especially if long-lasting. In addition, during the pandemic, people with mental conditions may find obstacles to accessing continuous treatment, including regular access to drugs and medical checks. As happened in Italy, people residing in nursing homes can be particularly at risk of abandonment with inadequate resources, overwhelmed staff and lack of contact with family members. People who report to emergency departments with suicidal behaviours may also be disadvantaged due to lengthy cues with priority given to suspected Covid-19 cases, resulting in suboptimal levels of care, which can potentially affect suicide numbers. As happened with the economic crisis between 2008-2010, it is particularly feared that the Covid-19 pandemic will provoke a global recession, capable of causing a significant number of 'economic suicides' through mechanisms such as loss of employment and indebtedness, and housing insecurity. 


\section{Zero Suicide: A Big Hairy Audacious Goal}

Joe Rafferty

Mr. Joe Rafferty is one of the co-founders of the Zero Suicide Alliance, a national suicide prevention collaboration of over 1500 members across the UK and the globe. Before coming to Mersey Care, which hosts the ZSA, Joe held the post of Director of Commissioning Support at the NHS Commissioning Board, having national responsibility for the design and delivery of a significant component of the commissioning side reforms outlined in the White Paper: Liberating the NHS. Prior to this, he was seconded from the Chief Executive role at NHS Central Lancashire, to become the Director of Commissioning Development for NHS North West, with a remit to lead the development of commissioning reforms across the North West of England. From 2007 to 2010 he was the Chief Executive of NHS Central Lancashire and the Regional Director of Commissioning and Strategy for NHS North West from 2006-2008. Other Board-level roles have included Director of Performance in Cumbria and Lancashire SHA and Director of System Reform at Bolton Hospital NHS Trust. Prior to these roles, he was part of the team that set up Greater Manchester SHA.

Abstract. This presentation explores the benefits of using audacious, zerobased thinking to stimulate change in healthcare provider and wider societal thinking in relation to suicide prevention. The Zero Suicide Alliance (ZSA) was born out of one mental health trust's thrust to begin to radically re-engineer it's thinking and delivery in relation to suicides of patients in their care. This approach of zero suicide created a focus and release of clinical creativity that has fundamentally alter the organisation's position on suicide prevention, it's approach to service redesign and its overall philosophy on learning - giving rise to a comprehensive and successful Just Culture programme. These benefits have become the centre point for establishing the Zero Suicide Alliance, a bottom up movement in the UK with a philosophy of knowledge into action. The presentation explores the benefits of the zero suicide approach and describes the work of the ZSA. 


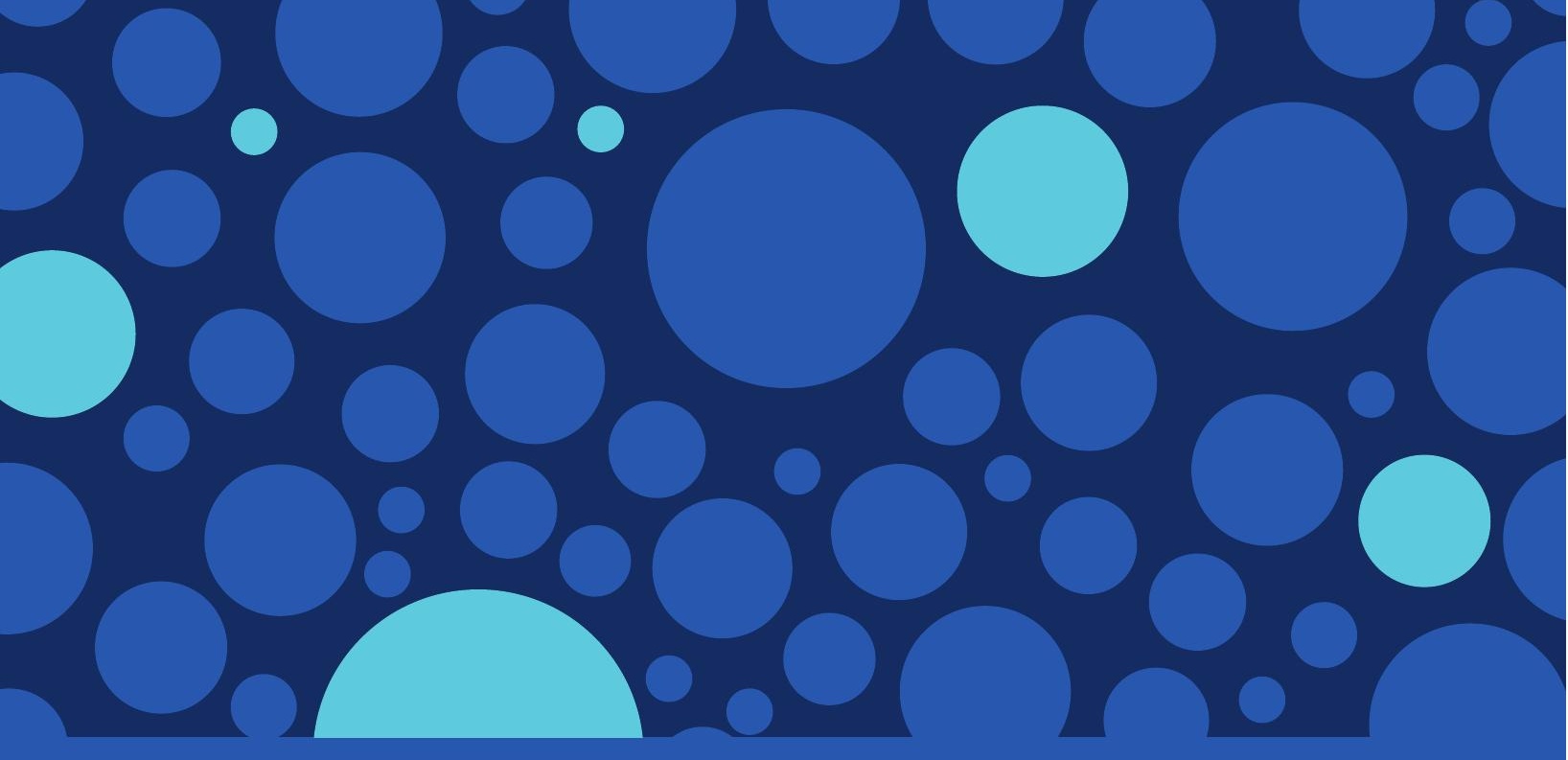

- I follow my intuition,

- I imagine more than ever,

- I love to innovate...

University of Primorska Press

www.hippocampus.si 